\title{
On the Temporal Granularity of Joint Energy-Reserve Markets in a high-RES system
}

\author{
Mathias Hermans ${ }^{1,2}$, Kenneth Bruninx ${ }^{1,2}$, Kenneth Van den Bergh ${ }^{1,2}$, Kris Poncelet ${ }^{1,2}$ \\ and Erik Delarue ${ }^{1,2, *}$ \\ ${ }^{1}$ Departement of Mechanical Engineering, KU Leuven, Leuven, Belgium \\ ${ }^{2}$ EnergyVille, Genk, Belgium
}

Abstract - The cost-efficient integration of variable renewable power (vRES) requires adequate operating reserve and energy markets. This paper focusses on the benefits of increasing the temporal granularity of joint energy-operating reserve markets in a case study on two high-vRES systems. Specifically, the impact of the frequency (i.e., how often reserves are sized and procured) and the resolution or contract duration (i.e., the length of the sizing and procurement blocks) is isolated by comparing year-long unit commitment and economic dispatch simulation results. We optimize the power system's operation in consecutive stages: scheduling of generation units with procurement of reserves, intra-day adjustments of generation schedules and real-time activation of flexibility to compensate for imbalances. The results suggest that dynamic and more frequent sizing, limiting the reserve procurement contract duration and a higher procurement frequency allow reducing the total operating cost by $1.5-1.8 \%$, while eliminating scarcity on reserve markets. These design changes facilitate the integration of intermittent renewables in reserve markets. Finally, some challenges following the proposed market design changes are highlighted.

Keywords - Balancing, operating reserves, reserve sizing, reserve procurement, renewables, unit commitment

* Corresponding author: prof. Erik Delarue. Email: erik.delarue@kuleuven.be. 


\section{Introduction}

Electricity generated by variable renewable energy sources (VRES), such as wind and solar PV, is variable in time and limitedly predictable by nature. Flexibility is required to compensate changes in renewable output and forecast errors. As the penetration of intermittent renewables increases, so may the need for flexibility and the development of associated markets.

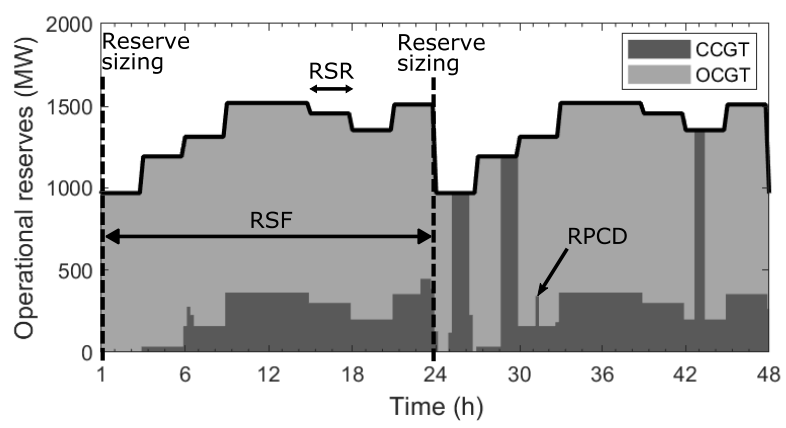

Fig. 1. Illustration of the upward reserve requirements and the temporal aspects of reserve markets, with a reserve sizing frequency (RSF) of $24 \mathrm{~h}$, reserve sizing resolution (RSR) of $3 \mathrm{~h}$ and reserve procurement contract duration (RPCD) of $1 \mathrm{qh}$. Reserve procurement (not shown) was synchronous with reserve sizing in this example. OCGT refers to open-cycle gas turbines, CCGT to combined-cycle gas turbines.

The transmission system operator (TSO) currently procures and deploys flexibility in the form of operating reserves. Three phases can be distinguished in this process: (i) the sizing or dimensioning of the needed reserve capacity, (ii) the procurement or allocation of reserves, and (iii) the activation of reserves in real-time. The temporal granularity of these reserve markets is determined by the frequency (i.e., how often reserves are sized and procured) and the resolution or contract duration (i.e., the length of the sizing and procurement blocks) (Fig. 1).

In today's European reserve markets, reserves (i.e., FCR, FRR and RR) are typically sized and procured on yearly, monthly, weekly or daily basis. Moreover, the contract duration for reserve products remains high in some countries [1]. However, a higher 
temporal resolution (e.g., daily sizing and procurement of hourly products) has the potential to lower the cost of reserves [2].

The focus of this paper is on the benefits regarding the cost-efficiency and scarcity on operating reserve markets associated with increasing their temporal granularity in highVRES power systems. In the scope of this work, we concentrate on automatic and manual FRR (typically referred to as operating reserve capacity in US markets). Concretely, we analyze the impact of the following four temporal aspects of reserve markets:

(1)The reserve sizing frequency (RSF) specifies how regularly reserves are sized.

(2)The reserve sizing resolution (RSR) sets the length of the sizing blocks.

(3)The reserve procurement frequency (RPF) sets how regularly reserves are procured.

(4)The reserve procurement contract duration (RPCD) specifies the resolution of reserve products, being allocated at unit level (i.e., length of the procurement blocks).

First, more frequent reserve sizing and procurement will allow employing improved forecasts as the lead time between forecast creation and actual realization decreases. Hence, the real-time operating reserve requirements (i.e., the reserve sizing) are reduced [3], which could yield operating cost savings while maintaining the system's security [4]. Second, increasing the temporal resolution of reserve markets allows more cost-efficient, dynamic sizing of reserves [2],[3]. Third, a short contract duration allows accounting for the time-dependent availability of reserve providers (particularly important for intermittent sources such as wind and solar PV, and certain types of responsive load or energy storage systems), as well as the time-dependent opportunity cost of offering capacity in the reserve markets.

A few authors [4]-[9] have studied the impact of the temporal granularity of energy- 
reserve markets on the cost-effectiveness of the resulting operating schedules, and focused on the frequency of sizing and procuring. Tuohy et al. [4] compare the performance of a stochastic and a deterministic scheduling method in a high-vRES test system inspired by the Irish power system and examine the influence of frequent rescheduling to account for updated wind and load forecasts. Hermans et al. [5] apply deterministic generation scheduling in a rolling horizon approach with various rescheduling frequencies to study the impact of the large-scale introduction of VRES on thermal power plant cycling behavior in a small scale test system. Jafari et al. [6] apply stochastic generation scheduling in a market model that explicitly incorporates a dayahead, intra-day and real-time balancing market to investigate the value of intra-day markets in a small test system with high wind power penetration. Schulze and McKinnon [7] propose improvements to the stochastic WILMAR model employed by Tuohy et al. [4], and explore the effects of pump storage and transmission restrictions on total cost for the British power system under National Grid's Gone Green scenario for 2020. Bucksteeg et al. [8] compare a novel dynamic cost-efficient reserve sizing method based on an explicit lead time dependent characterization of stochastic power system variables (such as vRES and load forecast errors) to a static sizing approach, and apply it with a deterministic daily (rolling horizon) rescheduling model on a hypothetical 2030 German power system. Dallinger et al. [9] investigate the impact of different reserve procurement contract durations and common procurement between control areas of a 2030 German power system scenario on total operating cost, $\mathrm{CO}_{2}$ emissions and VRES curtailment using the deterministic EDisOn+Balancing scheduling model.

Table I contrasts the considered changes to operating reserve market designs in each 
of the above references to identify the knowledge gap in the literature addressed by the present work. First, the impact of the reserve product contract duration and reserve sizing resolution has, to the best of the authors' knowledge, only been considered in [9]. Second, the scope of all the aforementioned literature was limited to a few different reserve procurement and market clearing frequencies (ranging from $3 \mathrm{~h}$ to $24 \mathrm{~h}$ ). In addition, the stochastic model formulations presented in [4],[6]-[7] implicitly ensure sufficient flexibility in the schedule, which implies the coupling of the reserve sizing and reserve procurement frequencies. In this work, we disconnect these frequencies and separate reserve sizing from reserve procurement entirely, mimicking European reserve markets. Third, in many European countries, the TSO dimensions the reserves based on a predefined reliability criterion that relates to the statistical description of observed imbalances. Such a criterion was not applied in the deterministic models of [5] and [7], in which the reserves were sized proportional to the forecasted VRES, nor in [9] where the reserve sizing problem was not considered.

In this work, the above-mentioned benefits of reducing the temporal granularity of reserve markets are quantified by leveraging a unit commitment and economic dispatch (UC) model, as in [4]-[9], presented in [14] and inspired by [15], explicitly considering a reliability criterion. Although a stochastic equivalent potentially leads to more costeffective operating schedules, the presented formulation ensures that the procured reserves are compliant with a predefined reliability criterion without requiring a large number of scenarios to accurately represent the range of possible imbalances [4],[6],[7]. A rolling horizon approach is taken to capture the temporal evolution of wind power uncertainty, as well as the functioning of joint energy-operating reserve and balancing 
markets. The three phases of reserve markets are considered: (1) sizing of the need for reserves, (2) procurement or allocation of reserves at unit level and (3) the activation of reserves to compensate for system imbalances. The model mimics a perfectly competitive electricity market with a central dispatch, mimicking most US markets [10] and a few European markets (e.g. Greece, Hungary, Ireland, Italy, Northern Ireland and Poland [11]). Although in other European countries electricity is traded day-ahead bilaterally and through power exchanges, the market outcome of both market designs is very similar under perfect competition [12],[13]. Finally, as opposed to the hourly time resolution adopted in [4]-[9], a quarter-hourly time resolution is employed to adequately capture the flexibility needs of the system and the activation of balancing reserves, following [16]-[19] and as recommended by FERC for high-vRES systems [20].

TABLE I: OVERVIEW OF THE CONSIDERED CHANGES TO OPERATING RESERVE MARKET DESIGNS IN THE SCIENTIFIC LITERATURE AND IN THIS PAPER.

\begin{tabular}{|c|c|c|c|c|c|c|c|}
\hline Authors & $\begin{array}{c}\text { UC model } \\
\text { (Reserve } \\
\text { sizing) }\end{array}$ & $\begin{array}{l}\text { Reliability } \\
\text { criterion }\end{array}$ & RSF & RSR & RPF & RPCD & $\begin{array}{l}\text { Scheduling } \\
\text { time } \\
\text { resolution }\end{array}$ \\
\hline $\begin{array}{c}\text { Tuohy et al. } \\
\text { [4] }\end{array}$ & $\begin{array}{l}\text { Stochastic } \\
\text { (implicit) }\end{array}$ & No & $3 h-6 h^{a}$ & $1 \mathrm{~h}$ & $3 h-6 h^{a}$ & $1 \mathrm{~h}$ & $1 \mathrm{~h}$ \\
\hline $\begin{array}{l}\text { Hermans et } \\
\text { al. [5] }\end{array}$ & $\begin{array}{c}\text { Deterministic } \\
\text { (explicit) }\end{array}$ & No & $4 h-24 h^{a}$ & $1 \mathrm{~h}$ & $4 h-24 h^{a}$ & $1 \mathrm{~h}$ & $1 \mathrm{~h}$ \\
\hline Jafari et al. [6] & $\begin{array}{c}\text { Stochastic } \\
\text { (implicit) }\end{array}$ & No & $6 h-24 h^{a}$ & $1 \mathrm{~h}$ & $6 h-24 h^{a}$ & $1 \mathrm{~h}$ & $1 \mathrm{~h}$ \\
\hline $\begin{array}{l}\text { Schulze and } \\
\text { McKinnon [7] }\end{array}$ & $\begin{array}{c}\text { Stochastic } \\
\text { (implicit) }\end{array}$ & No & $3 h-24 h^{a}$ & $1 \mathrm{~h}$ & $3 h-24 h^{a}$ & $1 \mathrm{~h}$ & $1 \mathrm{~h}$ \\
\hline $\begin{array}{c}\text { Bucksteeg et } \\
\text { al. [8] }\end{array}$ & $\begin{array}{c}\text { Deterministic } \\
\text { (explicit) }\end{array}$ & Yes & $24 \mathrm{~h}$ & $1 \mathrm{~h}$ & $24 \mathrm{~h}$ & $1 \mathrm{~h}$ & $1 \mathrm{~h}$ \\
\hline $\begin{array}{c}\text { Dallinger et } \\
\text { al. [9] }\end{array}$ & $\begin{array}{c}\text { Deterministic } \\
\text { (explicit) }\end{array}$ & No & Static & Static & $24 h-168 h$ & $4 h-84 h^{b}$ & $1 \mathrm{~h}$ \\
\hline This paper & $\begin{array}{c}\text { Deterministic } \\
\text { (explicit) }\end{array}$ & Yes & $1 q h->>36 h^{c}$ & $1 q h->>36 h^{c}$ & $1 q h-24 h$ & $1 q h-24 h$ & $1 q h$ \\
\hline
\end{tabular}


In summary, this work contributes to the existing literature by focusing on the interaction of (1) higher reserve sizing frequencies, which allows carrying less operating reserve capacity, (2) higher reserve procurement frequencies, enabling using more accurate vRES forecasts, (3) finer reserve sizing resolutions, allowing more cost-efficient, dynamic sizing of reserves, and (4) reduced procurement contract durations, allowing more cost-efficient schedules, a more diverse pool of reserve capacity providers and avoiding scarcity on reserve markets. We study the impacts of the temporal granularity of joint energy-operating reserve markets on operating costs and reserve market scarcity (as a measure for liquidity) in high-RES systems through the application of a state-of-the-art UC model in a rolling horizon approach (in accordance with [4]-[7]). By decoupling reserve product contract duration and reserve sizing resolution, explicitly varying each of the four identified temporal characteristics of reserve markets and by focusing on their interaction, we broaden the scope of existing literature.

This study is particularly relevant to European electricity markets, where the temporal granularity of energy and reserve markets are not coupled and TSOs explicitly set reserve requirements. We present a case study at scale on two high-RES systems. The results of our case studies reveal total operating cost reductions of $1.5 \%$ to $1.8 \%$ when adopting a higher temporal granularity in joint energy-reserve markets, primarily driven by decreased wind power uncertainty with lower forecasting lead times, which allows more cost-efficient reserve procurement. In this analysis, we explicitly show how much of these savings may be attributed to the four identified temporal resolutions of reserve markets. For Independent System Operators (ISOs), who typically operate joint dayahead operating reserve-energy markets with short reserve contract durations and high 
reserve sizing resolutions, the presented analysis sheds lights on the attainable cost benefits associated with higher reserve sizing and procurement frequencies.

This paper proceeds as follows. Section 2 presents the developed methodology and the test system for the case study. Section 3 presents and discusses the simulation results. Last, Section 4 concludes.

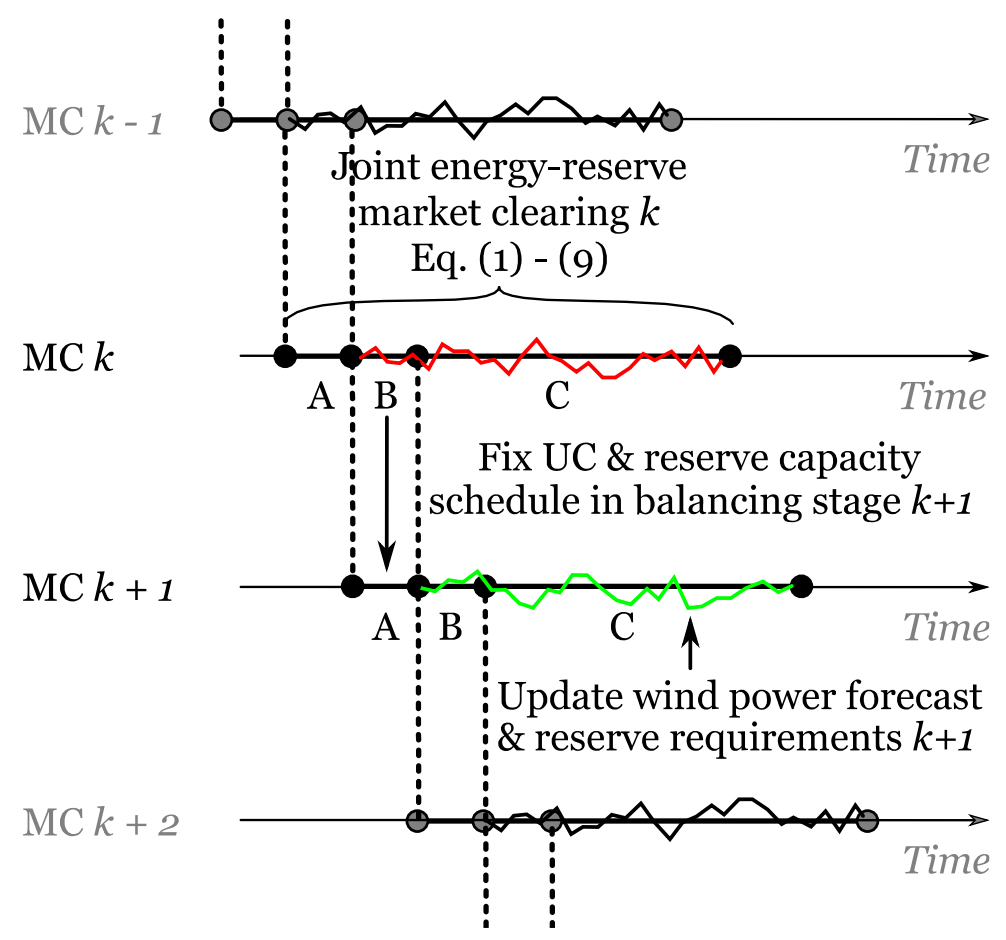

Fig. 2. The planning horizon of each Market Clearing Problem (MC) (Eq. (1)-(9)) consists of a balancing stage $A$, a final commitment stage $B$ and an update stage $C$. In the balancing stage $A$, the unit commitment and reserve capacity schedule is fixed based on the results on the final commitment stage $B$ in the previous market clearing problem. For each Market Clearing Problem the wind power forecast and reserve requirements are updated based on the last available observation (Section 2.2). The dispatch results in all balancing periods (A) and start-up costs incurred in all final commitment stages (B) jointly determine the total operating costs (Section 3.1). Similarly, real-time emergency measures deployed during balancing periods $(A)$ and flexible load scheduled as an emergency measure during the final commitment stage (B) are used to gauge reserve capacity market scarcity (Section 3.2).

\section{Methodology}

The rolling horizon approach (Fig. 2) allows modeling joint energy-reserve markets and real-time balancing markets. By splitting the total simulation period into overlapping planning loops, the market clearing and reserve procurement frequency is represented, 
and adjustments to the unit commitment schedule are allowed as the model is provided with updated wind power forecasts. The horizon of each planning loop consists of a balancing stage $A$, a final commitment stage $B$ (representing the period for which the joint energy-reserve market is cleared and setting the energy-reserve market clearing frequency, RPF) and an update stage C (Fig. 2). The commitment statuses in stage B and $\mathrm{C}$ are allowed to be adjusted with respect to the previous loop, as long as all technical limitations are respected. The wind power uncertainty, and hence the forecasting accuracy, depends on the lead time since the first time step of stage B. The commitment decisions made in stage $B$ are carried over to the balancing stage $(A)$ of the next market clearing, where they are fixed. While the schedule in stages $B$ and $C$ is subject to reserve requirements and wind power forecast errors, the actual wind power generation is assumed to be known in the balancing stage $(A)$ and the reserve requirements are lifted, representing the activation of the procured reserves in the balancing market. For the purpose of this study, the expected activation cost of reserve providers is not considered in the allocation phase (stages B and C in Fig. 2), but the effective activation costs are accounted for in the balancing stage $A$.

Below, we first discuss the employed unit commitment model. Second, the dedicated wind power forecast scenario generation tool is briefly described. Finally, we describe the test systems for the case studies.

\subsection{Unit Commitment Model Description}

We extend the UC model formulation from [14], formulated as a mixed integer linear program, inspired by [15]. More specifically, we introduce a set of constraints to impose a reserve procurement contract duration. 
In the objective function, the operating cost under forecast conditions (fuel costs $c_{i, t}^{F}$, start-up costs $c_{i, t}^{S U}$, ramping costs $c_{i, t}^{R}, \mathrm{CO}_{2}$-emission costs $c_{i, t}^{C O 2}$ and the cost of loadshedding $\lambda^{\Phi} \cdot \Phi_{t}$ ) are complemented with a proxy for the cost associated with reserve shedding in the commitment stage B and update stage C:

$\min \sum_{j}\left(\sum_{i}\left(c_{i, t}^{S U}+c_{i, t}^{F}+c_{i, t}^{C O 2}+c_{i, t}^{R}\right)+\lambda^{\Phi} \cdot \Phi_{t}\right)+\sum_{t} \lambda^{\Phi} / 2 \cdot \Phi_{t}^{+}$

Reserve shedding implies that emergency measures are activated to safeguard system security, such as shedding of load, and is penalized at the half of the value of lost load (VOLL, $\left.\lambda^{\Phi}\right)$. As such, the model prioritizes the use of emergency measures to meet the reserve requirements instead of the expected load (compliant with Article 152(8) of Commission Regulation (EU) 2017/1485 [21]). This allows hedging the operating schedule against overly conservative reserve requirements, in which the costs of providing reserves would exceed their benefits (i.e., avoiding the deployment of these emergency measures). Being a last-resort option due to the high cost of the emergency measures, reserve shedding indicates scarcity on the reserve market, which may be interpreted as a measure of liquidity.

At each time step $t \in T$, the demand for electricity $\left(D_{t}\right)$ minus load curtailment $\left(\Phi_{t}\right)$ must match the supply of electricity from conventional power plants $i \in I\left(g_{i, t}\right)$ and renewable energy sources $\left(G_{t}^{F}\right)$, corrected for curtailment $\left(\chi_{t}\right)$ under forecast conditions, which is assumed to be free:

$\forall t: D_{t}-\Phi_{t}=\sum_{i} g_{i, t}+G_{t}^{F}-\chi_{t}$

In what follows, we briefly discuss the operating reserve requirements. Subsequently, we present the taken approach to introduce varying reserve sizing resolutions and contract durations. For sake of brevity, we do not discuss technical constraints, such as 
binary logic constraints, generation limits, upward/downward ramping limits $(R U / R D)$ and minimum up and down time (MUT/MDT) constraints. A full description of these constraints can be found in [14],[22]. Note that we do not consider transmission grid constraints in our analysis. Furthermore, this paper does not aim to assess the effectiveness of reserve deployment. We assume that the contractual obligations and requirements regarding deployment of these operating reserves are fulfilled by those generating units scheduled to provide them. Furthermore, the maximum ramp up and down rates of the units are considered by the UC model while scheduling the required reserve capacity, following [14].

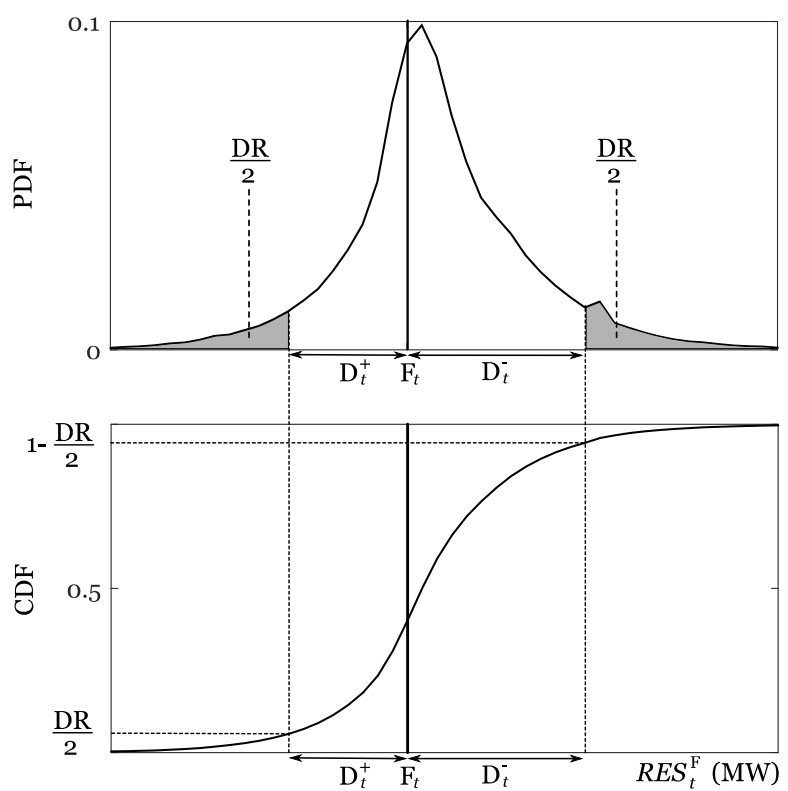

Fig. 3. The design reliability (DR) is the cumulative probability of forecast errors that are not covered by the operating reserves. The DR is equally distributed over the upward and downward reserves. Using the cumulative probability density function of the wind power forecast errors (at the forecast lead time on each time step $t$ ), the corresponding upward $D_{t}^{+}$and downward reserve requirements $D_{t}^{-}$can be determined relative to the central forecast $F_{t}$.

\subsubsection{Operating reserve requirements}

A statistical analysis of observed wind power forecast errors, which is assumed to be the only source of uncertainty and is further detailed in Section 2.2, yields probability 
density functions of the forecast errors. Starting from this statistical description, reserve requirements can be sized to cover a predetermined percentile range of possible forecast errors at each time step, as stipulated by a reliability criterion, i.e., a design reliability. The procedure of setting the upward and downward reserve requirements based on a design reliability is visualized in Fig. 3. While doing so, we account for the reserve sizing frequency and reserve sizing resolution. When the reserve sizing resolution exceeds one time period, the most conservative dimensioning within each block period is enforced for the whole period. For example, when the reserves are sized daily with a 24 hour resolution, then the highest wind power forecast uncertainty in the coming 24 hours determines the reserve requirement for the whole day.

At each time step, the upward and downward reserve requirements must be satisfied:

$\forall t: \quad D_{t}^{+}=\sum_{i} r_{i, t}^{+}+n s r_{i, t}^{+}+\chi_{t}^{+}+\Phi_{t}^{+}$

$\forall t: \quad D_{t}^{-}=\sum_{i} r_{i, t}^{-}+\chi_{t}^{-}$

Aside from spinning $\left(r_{i, t}^{+}\right.$and $\left.r_{i, t}^{-}\right)$or non-spinning reserves $\left(n s r_{i, t}^{+}\right)$provided by conventional generating units, the disconnection of flexible load $\left(\Phi_{t}^{+} \geq 0\right)$ and curtailment of RES-based generation $\left(\chi_{t}^{-}, \chi_{t}^{+} \geq 0\right)$ are explicitly considered as flexibility options. RES-based generation as upward reserve provider is limited to the scheduled curtailment $\left(\chi_{t}^{+} \leq \chi_{t} \leq G_{t}^{F}\right)$.

\subsubsection{Reserve procurement contract duration}

The reserve procurement contract duration $(R P C D)$ is imposed at unit level through equations (5)-(9). They impose that the amount of upward and/or downward reserves provided by each unit or RES remains constant across adjacent blocks of equal duration, set by $R P C D$ : 


$$
\begin{aligned}
& \forall t \in(t \bmod R P C D>0): \\
& r_{i, t}^{+}=r_{i, t+1}^{+} \\
& n s r_{i, t}^{+}=n s r_{i, t+1}^{+} \\
& \chi_{t}^{+}=\chi_{t+1}^{+} \\
& r_{i, t}^{-}=r_{i, t+1}^{-} \\
& \chi_{t}^{-}=\chi_{t+1}^{-}
\end{aligned}
$$

\subsection{Wind Power Forecast Scenario Generation}

To study the impact of the temporal granularity of reserve markets, it is essential to consider the relationship between forecast errors and forecasting horizon. Therefore, we employ a dedicated wind power forecast generation tool, presented in [23]. This data-driven tool allows generating forecast updates that mimic the accuracy of today's forecasting tools. Below, we briefly describe its working principles and performance. A full description can be found in [23].

A statistical analysis of day-ahead and intra-day wind power forecasts and measured wind power data from 2016 to 2018 for Belgium [24] confirmed three crucial characteristics of wind power forecast errors in the context of this paper:

(1) The accuracy of wind power forecasts improves as the forecast lead time (the time between forecast creation and actual realization) decreases;

(2) The observed forecast errors are limited to a range around the forecast error in the previous (quarter-hourly) time step, which in turn is dependent on the measured power.

(3) The day-ahead and intra-day forecasts were positively correlated.

To ensure these aspects are reflected in the generated forecast updates, we sorted 
the historically observed forecast errors based on a combination of lead time, measured power interval and interval of the forecast error in the previous time step. We calculated a probability density function (PDF) for each of the groups of forecast errors assigned to the observed combinations. In addition, we calculated the correlation coefficients between all lead times observed in the two time series to build a correlation matrix $\Sigma^{i}$. Next, forecasts are generated as disturbances (errors) to the measured wind power (historical data). For the target forecast time, a vector of random samples $N\left(0, \Sigma^{i}\right)$ is drawn from the standard multivariate normal distribution, representing normalized forecasts with different lead times. These are then transformed to wind power forecast error scenarios via the PDFs acquired in the statistical analysis, following [20]. This process is repeated for each time step of the simulation period.

A series of quarter-hourly forecast updates is plotted in Fig. 4. In [23], we show that the mean absolute percentage error, the error quantiles and the correlation coefficients of generated forecasts closely approximate those of historical forecast data. As such, the generated forecasts comply with the three characteristics discussed above.

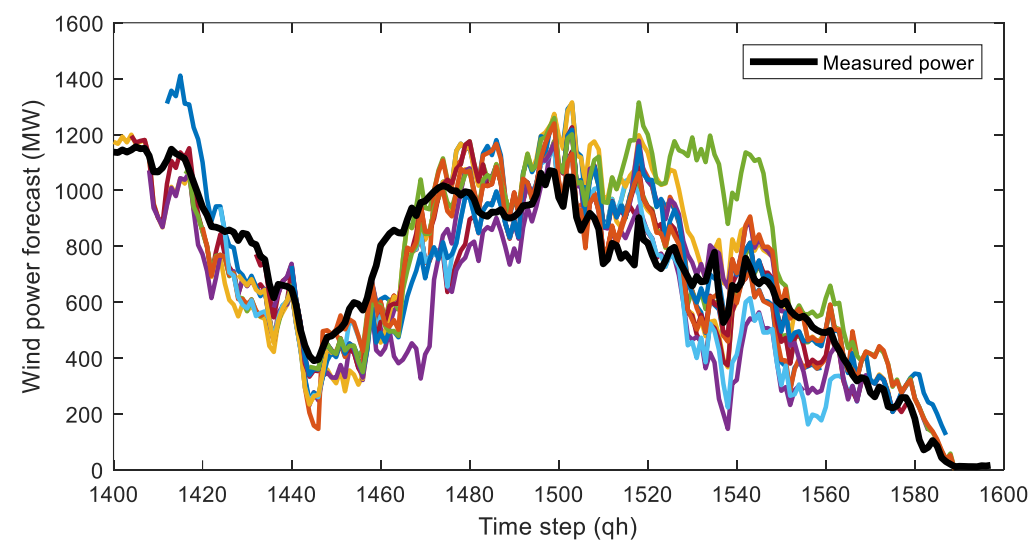

Fig. 4. Superimposing the generated forecast errors on a central forecast (the measured power, black line) yields forecast updates (hourly updates shown in color, which each color indicating a forecast update associated with another time step). 


\subsection{Test Systems}

The test systems for our case studies originate from two scenarios for Belgium in the context of the EU-SysFlex project [25]. They will be referred to as the Energy Transition (ET) and the Renewable Ambition (RA) scenarios. Table I provides an overview of their associated conventional generation portfolios and the technical characteristics of their generation units, taken from [26]-[34]. The total thermal capacity in the ET and RA systems amount to $11.2 \mathrm{GW}$ and $17.8 \mathrm{GW}$, with the latter consisting of more flexible gas-fired capacity. The OCGT units are pooled into one single unit. All CCGT units can provide spinning reserves, and the OCGT can provide non-spinning reserves. The pumped hydro storage unit has a $5.8 \mathrm{GWh}$ energy capacity in both systems.

Power plant generation is scheduled for a full year. Quarter-hourly measured wind and solar power output, as well as load time series from the year 2018 are taken from Elia [24]. The wind and solar power output (and capacities) were rescaled (keeping the original ratio between onshore and offshore wind) such that they correspond to solar power capacities of $3.8 \mathrm{GW}$ and $4.7 \mathrm{GW}$ and wind power capacities of $6.9 \mathrm{GW}$ and 9.3 GW in the ET and RA scenarios. Cogeneration, biomass and hydro power yearly profiles

for 2013 were taken from [35] and scaled to match the aggregated annual data from the EU-SysFlex scenarios. The total RES-based generation amounts to $31 \%$ of total electricity demand (ET: 98 TWh and RA: 119.1 TWh) in both scenarios. Renewables curtailment is assumed to be free of cost and the socio-economic cost of load shedding is assumed to be $10,000 € / \mathrm{MWh}$. Upon reserve sizing, the upward and downward reserves at each time step are sized to cover a 99 percentile range of possible upward and downward imbalances caused by wind power forecast errors, as set by the Belgian 
TABLE I: Energy Transition (ET) and Renewable Ambition (RA) thermal and pumped hydro generation portfolios (SPP: steam power plant)

\begin{tabular}{|c|c|c|c|c|c|c|c|c|c|c|}
\hline Type $^{d}$ & Un & $\mathrm{s}[\#]$ & $\begin{array}{l}\text { Unit } \\
\text { size } \bar{P} \\
{[\mathrm{MW}]}\end{array}$ & $\begin{array}{c}\text { Efficie } \\
\text { ncy } \\
{[\%]}\end{array}$ & $\begin{array}{l}\frac{P}{\min } \\
{[\% \bar{P}]}\end{array}$ & $\begin{array}{l}\text { Start- } \\
\text { up } \\
\text { rate } \\
{[\% \overline{\mathrm{P}} / \mathrm{q}} \\
\mathrm{h}]\end{array}$ & $\begin{array}{l}\text { Ramp } \\
\text { up/ } \\
\text { down } \\
\text { rate } \\
{[\% \bar{P} /} \\
\text { qh] }\end{array}$ & $\begin{array}{c}\text { MDT/ } \\
\text { MUT } \\
{[h]}\end{array}$ & $\begin{array}{l}\text { Start- } \\
\text { up } \\
\text { cost } \\
{[€ / M} \\
\text { W/ } \\
\text { start] }\end{array}$ & $\begin{array}{c}\text { No } \\
\text { load } \\
\text { cost }^{b} \\
{[€ / M} \\
\text { W/qh] }\end{array}$ \\
\hline SPP-oil & 2 & 0 & $40-82$ & 40 & 43 & 48 & 9 & $5 / 5$ & 134.75 & 25 \\
\hline SPP-bio & 1 & 1 & $\begin{array}{l}800- \\
500^{a}\end{array}$ & 46 & 43 & 54 & 23 & $6 / 6$ & 134.75 & 2 \\
\hline $\begin{array}{l}\text { CCGT- } \\
\text { existing- } \\
\text { old }\end{array}$ & 3 & 3 & $\begin{array}{l}464- \\
475\end{array}$ & 48 & 35 & 58 & 45 & $2 / 4$ & 56.2 & 7.7 \\
\hline $\begin{array}{l}\text { CCGT- } \\
\text { existing- } \\
\text { new }\end{array}$ & 3 & 3 & $\begin{array}{l}420- \\
430\end{array}$ & 58 & 35 & 58 & 45 & $2 / 4$ & 56.2 & 6.4 \\
\hline $\begin{array}{l}\text { CCGT- } \\
\text { expansio } \\
\text { n-new }\end{array}$ & 12 & 22 & 540 & 58 & 35 & 58 & 45 & $2 / 4$ & 56.2 & 6.4 \\
\hline OCGT & 1 & 1 & $\begin{array}{l}1158- \\
2700^{a}\end{array}$ & 42 & 0 & 100 & 100 & $\begin{array}{c}0.25 / 0 \\
.25\end{array}$ & 0 & 1.1 \\
\hline $\begin{array}{c}\text { Pumped } \\
\text { hydro } \\
\text { storage }\end{array}$ & 1 & 1 & 1308 & $0.74^{c}$ & 0 & 100 & 100 & $5 / 5$ & 0 & 0 \\
\hline \multicolumn{11}{|c|}{$\begin{array}{l}{ }^{\mathrm{a}} \text { Capacity in ET and RA portfolio (existing and capacity expansion). } \\
\text { b Per MW rated capacity. } \\
{ }^{\mathrm{c}} \text { Roundtrip charging-discharging efficiency. } \\
{ }^{\mathrm{d}} \text { The fuel prices in the ET and RA scenarios are } € 28 \text { and } € 15 / \mathrm{MWh} \text { th for natural gas, } € 73.80 \text { and } \\
€ 61.43 / \mathrm{MWh}_{\text {th }} \text { for oil, and } € 6 \text { and } € 6 / \mathrm{MWh} \text { th for biomass. The emission allowance prices are } 27 \text { and } \\
90 € / \mathrm{tCO}_{2} \text {. }\end{array}$} \\
\hline \multirow{2}{*}{$\begin{array}{c}\text { Temporal } \\
\text { aspect }\end{array}$} & \multicolumn{10}{|c|}{ Cases from least (1) to most (6) granular } \\
\hline & & (1) & & $(2)$ & (3) & & $(4)$ & $(5)$ & & $(6)$ \\
\hline RSF & & $36 h^{a}$ & $\rightarrow$ & $24 \mathrm{~h}$ & $24 \mathrm{~h}$ & & $24 \mathrm{~h}$ & $24 \mathrm{~h}$ & $\rightarrow$ & $1 \mathrm{~h}$ \\
\hline RSR & & $36 h$ & $\rightarrow$ & $24 \mathrm{~h}$ & $24 \mathrm{~h}$ & $\rightarrow$ & $1 q h$ & $1 \mathrm{qh}$ & & $1 q h$ \\
\hline RPF & & $4 \mathrm{~h}$ & & $24 \mathrm{~h}$ & $24 \mathrm{~h}$ & & $24 \mathrm{~h}$ & $1 \mathrm{~h}$ & & $1 \mathrm{~h}$ \\
\hline RPCD & & $4 \mathrm{~h}$ & & $24 \mathrm{~h}$ & $1 q h$ & & $1 q h$ & $1 \mathrm{qh}$ & & $1 q h$ \\
\hline
\end{tabular}

a Taking into account wind power uncertainty associated with the maximum historically observed forecast lead times and 36-37 hours.

TSO [36] compliant with Article 157(h) in [21]. Figure 1 shows an example of the dynamic upward reserve requirements (RR). Import and export time series were taken from a day-ahead scheduling simulation of the Central Western European (CWE) electricity 
market in which reserves were not considered. To ensure computational tractability, a complete CWE system with transmission network constraints was not considered.

For each case, the generation schedule and reserves allocation is optimized yielding yearlong schedules of which the expected values are reported. The unit commitment and economic dispatch will quantify the total operating system cost for scenarios with different frequencies and resolutions or contract durations for the sizing and procurement of reserves. Out of the myriad of possible combinations of the four considered aspects of temporal granularity, six base sets were chosen, as outlined in Table II. These set-ups range from very conservative to highly dynamic such that clear insights could be gained on the impact of the temporal resolution of each aspect of the reserve markets.

The MIP optimality tolerance is set at $0.25 \%$. The UC simulations were run on the Genius High Performance Computing cluster of the KU Leuven, using $2.3 \mathrm{GHz}$ computing nodes with 36 cores and 192 GB of RAM. Mostly depending on the clearing frequency, computation times ranged from 12 days (for an hourly market clearing frequency) to 3.5 hours (for a 24-hourly market clearing frequency).

\section{Results \& discussion}

We present the evolution of the total operating cost across the six cases of temporal granularity in Section 3.1. We do the same for the impact on scarcity in the reserve market in Section 3.2. Finally, we reflect on our methodology and findings in relation to current European market operation. 


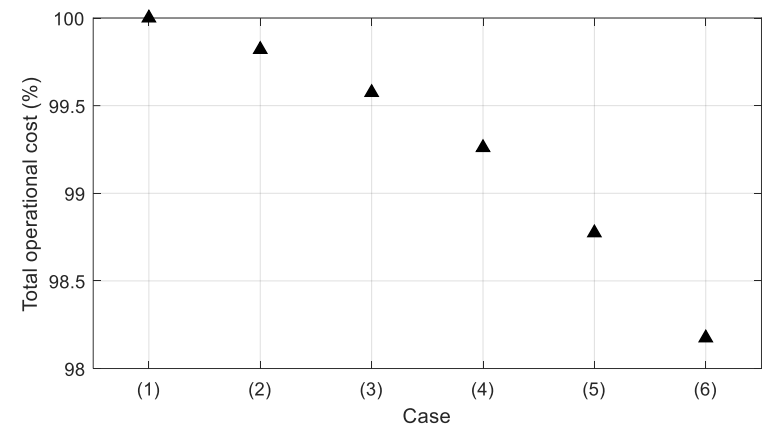

Fig. 5. Operating cost savings (normalized w.r.t. the total operating cost for case (1)) are at most $1.8 \%$ (case 6 ) by adopting the highest temporal granularityin the ET system.

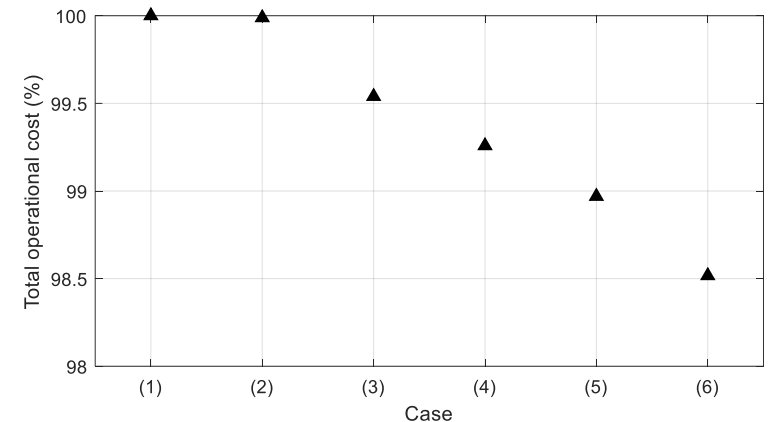

Fig. 6. Operating cost savings (normalized w.r.t. the total operating cost for case (1)) are at most $1.5 \%$ (case 6) by adopting the highest temporal granularity in the RA system.

\subsection{Impact of temporal granularity on total operating cost}

Figures 5 and 6 show the total operating costs in the six cases for the ET and RA systems. The breakdown of the operating costs, reserve procurement and reserve activation presented in Table III, supports the discussion of the cost differences given below. We find that in both systems, the general trend is a total operating cost decrease when moving from the most conservative (case 1) to a high temporal granularity (case 6), resulting in a total cost difference of $1.8 \%$ and $1.5 \%$ of total operating costs for the ET and RA systems. These estimated cost reductions are in line with findings in the scientific literature. For example, Dallinger et al. [9] report total operating cost savings of $0.6 \%$ by moving to a daily procurement (reserve sizing and procurement frequency of 
24 hours) of four hour reserve products (reserve product contract duration and reserve sizing resolution equal to 4 hours) based on a case study considering a hypothetical 2030 German power system.

By far, the largest cost savings are the result of sizing the reserves more frequently. While the impact of moving from yearly to daily sizing (cases $1-2)$ is modest $(0-0.2 \%)$, the cost savings obtained by further increasing the sizing frequency to an hourly (cases $5-6)$ rate reach $0.6 \%$ and $0.45 \%$ for the ET and RA systems. The main driver of these cost savings is the reduced wind power uncertainty associated with shorter forecasting lead times. Consequently, the final planning is made under less wind power forecast uncertainty as the reserves are procured more frequently. The reduced uncertainty space requires the procurement and activation of less reserve capacity, as shown in Table III. This saves costs in two ways. First, generation costs decrease, as carrying less spinning reserves requires less part-load operation. Additionally, upholding less nonspinning reserves saves OCGT no load costs (and thus, generation costs) as they are less often in stand-by. Second, fewer CCGTs are required to meet demand, leading to fewer start-ups and associated costs. Although reserve activation costs (not listed in Table III) increase as the OCGTs play a more prominent role in balancing, they only slightly offset the generation and start-up cost savings. Note that, since in the RA system all reserves are provided by the OCGT capacity in case 1 , it is impossible to reduce CCGT participation and associated costs when moving to daily sizing (case 2). Consequently, there are no total operating cost savings from case 1 to case 2 . Completely omitting the 24-hour reserve procurement contract duration (cases $2-3$ ) yields $0.25 \%$ and $0.45 \%$ cost savings for the ET and RA systems. 
TABLE III: Breakdown of operating costs, reserve procurement and reserve activation (RP: reserve procurement, RA: reserve activation) ${ }^{a}$

\begin{tabular}{ccccccc}
\hline \hline Energy Transition & \multicolumn{7}{c}{ Case } & $(2)$ & $(3)$ & $(4)$ & $(5)$ & $(6)$ \\
\hline Upw. RR (MW) & 1606 & 1517 & 1517 & 1217 & 1217 & 805 \\
Upw. RP CCGT (MW) & 451 & 362 & 599 & 357 & 487 & 373 \\
Upw. RP OCGT (MW) & 1155 & 1155 & 947 & 882 & 764 & 473 \\
Upw. RP RES (MW) & 0.2 & 0.0 & 2.4 & 2.0 & 1.8 & 1.3 \\
Upw. RA (MW) & 172 & 172 & 173 & 173 & 111 & 111 \\
Upw. RA CCGT (MW) & 152 & 144 & 145 & 103 & 82 & 67 \\
Upw. RA OCGT (MW) & 18 & 26 & 26 & 68 & 28 & 42 \\
Upw. RA RES (MW) & 1.4 & 1.2 & 2.0 & 1.6 & 1.2 & 0.8 \\
Generation cost (M€) & 2414.9 & 2410.2 & 2405.2 & 2396.0 & 2403.8 & 2396.8 \\
Start-up cost (M€) & 56.7 & 56.9 & 55.4 & 56.4 & 35.5 & 27.5 \\
Total operating cost (M€) & 2475.8 & 2471.4 & 2465.3 & 2457.5 & 2445.5 & 2430.6 \\
\hline Renewable Ambition & $(1)$ & $(2)$ & $(3)$ & $(4)$ & $(5)$ & $(6)$ \\
\hline Upw. RR (MW) & 2170 & 2049 & 2049 & 1645 & 1645 & 1088 \\
Upw. RP CCGT (MW) & 0 & 0 & 652 & 703 & 844 & 763 \\
Upw. RP OCGT (MW) & 2191 & 2070 & 1469 & 1024 & 880 & 411 \\
Upw. RP RES (MW) & 0.0 & 0.0 & 2.0 & 2.1 & 1.6 & 1.6 \\
Upw. RA (MW) & 247 & 246 & 250 & 251 & 154 & 153 \\
Upw. RA CCGT (MW) & 100 & 99 & 117 & 131 & 100 & 117 \\
Upw. RA OCGT (MW) & 146 & 146 & 132 & 118 & 52 & 34 \\
Upw. RA RES (MW) & 1.1 & 1.1 & 1.2 & 1.4 & 0.9 & 0.9 \\
Generation cost (M€) & 4475.7 & 4475.9 & 4461.7 & 4453.9 & 4461.1 & 4440.1 \\
Start-up cost (M€) & 75.1 & 74.3 & 67.0 & 61.0 & 41.0 & 42.0 \\
Total operating cost (M€) & 4558.7 & 4558.1 & 4537.7 & 4524.8 & 4511.7 & 4491.0 \\
\hline \hline
\end{tabular}

${ }^{a}$ Mean procurement or activation over all time steps of the year.

This decrease is solely driven by procurement efficiency gains. The unused capacity of CCGTs operating at part-load during the mid-day demand valley can now partly replace the OCGT capacity in the reserve provision (Table III), driving down the OCGT no load costs. Furthermore, it allows wind power to provide inexpensive reserves for a short time (even for a quarter hour), which it would not have been able to provide at the same level for 24 hours. Hence, it facilitates the integration of intermittent renewables in reserve markets. Although it covers less than $0.2 \%$ of the total upward RR, all planned wind power curtailment is scheduled as upward reserves at a quarter-hourly reserve procurement contract duration (Table III).

Moving to high resolution reserve sizing (cases 3-4) entails a cost benefit of around 
$0.3 \%$ for both systems as it allows more dynamic dimensioning. Hence, the full advantage of the reduced wind power uncertainty associated with shorter forecasting lead times may be leveraged. As can be seen in Table III, this results in less conservative reserve requirements and lower operating costs. Note that the benefits of dynamic dimensioning can only be captured when sizing reserves with at least a daily frequency. As such, the forecasting lead times are 24 hours or less, below which wind power uncertainty decreases considerably.

Finally, introducing very frequent (e.g., hourly) reserve procurement without adopting more frequent reserve sizing (cases 4-5) reduces wind power uncertainty, but does not reduce reserve sizing accordingly. The frequent planning adjustments cause a shift of the real time balancing efforts to the planning stage, where it happens more costefficient through changes in commitment statuses. The resulting generation cost increase is more than compensated by reduced reserve activation costs, yielding $0.50 \%$ and $0.30 \%$ total cost savings.

Note that we compare the total operating costs after balancing, but exclude costs associated with possible load shedding. In every time step, the operating reserves are sized to cover at least a 99 percentile range of historical upward and downward imbalances due to forecast errors, in line with current regulation [36],[21]. Therefore, even the full activation of the scheduled reserves would not suffice to balance forecast errors at the outer two percentiles of the probability distributions. The Belgian TSO can take exceptional measures to mitigate the remaining system imbalance after exhaustion of the available reserve capacity. They are described as "changes in the active power production or consumption of power generating modules and demand units" and may 
include the activation of slow start units [36]. Hence, we modeled these measures as real time load shedding to ensure that they are a last resort option. As the impact of these measures on the total operating cost is highly dependent on the assumed VOLL, the load curtailment costs are not included in the total operating costs discussed above.

\subsection{Impact of temporal granularity on reserve market scarcity}

In this paper, we assess the system's ability to procure the operating reserves while balancing supply and demand under forecast conditions. We gauge potential scarcity on the reserve market by the amount of flexible load scheduled as an emergency measure to provide energy or reserves in the final commitment stage B (Fig. 2). We distinguish such emergency reserve procurement from the real time emergency measures during balancing (RTEM). The latter are used to evaluate the system's reliability (and more specifically, operational security), as they include the exceptional measures taken by the TSO to mitigate the remaining system imbalance after exhaustion of the available reserve capacity. However, the TSO accepts the exceptional activation of such measures when setting the reliability criterion to $99 \%$. We have verified that the resulting schedules in all our case studies are compliant with the reliability criterion, even if scarcity is observed in the DA reserve capacity markets.

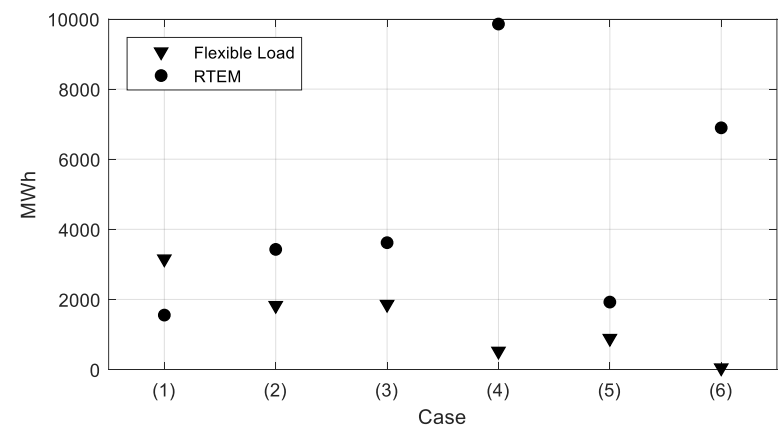

Fig. 7. The amount of load curtailment scheduled in the energy and/or reserve markets, as a measure of reserve market liquidity, in the ET scenario is completely eliminated by adopting the highest temporal granularity. 


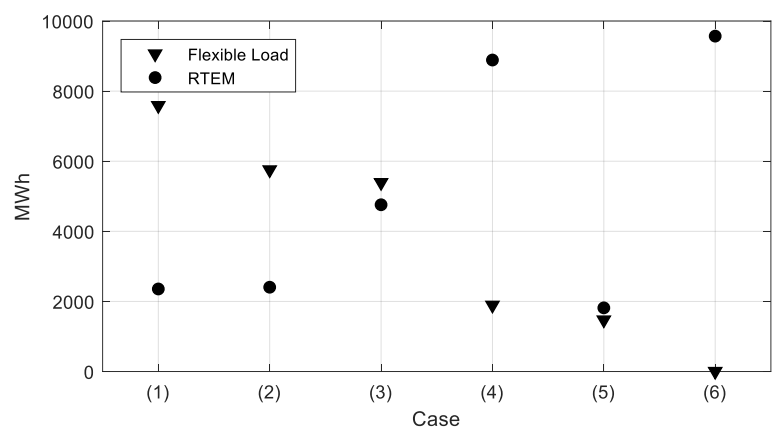

Fig. 8. The amount of load curtailment scheduled in the energy and/or reserve markets, as a measure of reserve market liquidity, in the RA scenario is completely eliminated by adopting the highest temporal granularity.

Figures 7 and 8 show the scheduled flexible load and the RTEM in the six cases for the ET and RA systems. The general trend is a flexible load decrease when moving from the most conservative (case 1) to a highly dynamic (case 6) temporal granularity, with a near elimination of scheduling flexible load. Reserve market scarcity reduces most notably with the adoption of more dynamic reserve sizing, both in terms of frequency (cases 12 and cases 5-6) and resolution (cases 1-2 and cases 3-4). More dynamic reserve sizing reduces over dimensioning of the reserve requirements. Note that eliminating the reserve procurement contract duration (cases 2-3) has no clear impact on flexible load in the ET system, and causes only a small improvement in the RA system.

The evolution of the RTEM is linked to the extent by which the reserves are over dimensioned in relation to the reliability criterion. More conservative reserve sizing (case 1) leads to low RTEM volumes. The RTEM peaks when the wind power uncertainty captured by the reserve sizing equals the uncertainty under which the final planning is made. This is the case when reserve sizing coincides with procurement (cases 4 and 6). Note, however, that also in this case, the ex-ante reliability criterion is met in real time. 


\subsection{Relation to current European market operation}

This work quantifies the potential benefits to cost-efficiency (and hence welfare) and reserve market liquidity of adopting increasingly more dynamic temporal granularities of reserve markets. Implementing and operating these ever more complex reserve capacity markets may, however, induce direct (i.e., related to the management and clearing of the markets) and indirect (i.e., changes in reserve capacity bids and procurement volumes) costs. Below, we reflect on some of the practical challenges and considerations of market participants in the context of current European market:

- Hourly clearing of reserve markets (case 5 and 6 ) could pose timing difficulties related to shortening market players' lead times between receiving updated forecast information and acting on that information and communicating to the TSO. Furthermore, energy markets would have to be cleared equally frequent such that reserve procurement always precedes the energy market clearing. This prevents that all capacity able to participate in the reserve market would have possibly been sold in the energy market [37]. Among other practical difficulties, hourly reserve markets may amplify issues related to MUT/MDT restrictions and the allocation of start-up and no-load costs across market intervals. Indeed, the shorter clearing horizon implied by more frequent energy market clearing could require generator start-up costs to be spread over smaller bid energy quantities, which could drive up market clearing prices. Similarly, profitable market participation by pumped hydro storage units could be compromised, because the optimal use of linked blocks for bidding pump-turbine cycles may require market price valleys and peaks to fall within the clearing horizon. 
- While this work assumes that contract durations are imposed at unit-level, current European reserve market rules stipulate product resolutions on balancing service providers' (BSP) portfolio level. The BSPs can decide on the allocation of its assets (durations and capacities) to fill in his reserve obligations as long as all technical requirements such as activation times can be met. While this assumption is conservative, it is acceptable, since leveraging the flexibility of multiple technologies to assemble a single reserve bid at portfolio level is not without difficulties, especially for smaller and less diversified portfolios.

- We assume risk-neutral behavior of the TSO and market participants. The TSO might perceive more risk when reserves are contracted on a daily or hourly basis. Indeed, in case of low liquidity in reserve markets, the TSO would have considerably less time to find additional reserve providers to avoid scarcity in the reserve markets. Such concentrated reserve capacity markets may be prone to market power abuse, driving up reserve procurement costs [38]. On the other hand, BSPs are likely to be less risk averse towards offering in more frequent reserve markets as they would have a much better view on the availability of their asset (e.g., wind power forecasts are only reliable one or two days ahead) [35]. Therefore, it reduces their uncertainty on the volumes that could be sold in energy and reserve markets throughout the time during which that flexibility would be contracted. Allowing arbitrage between energy and reserve markets could eliminate the time-value premium on bidding flexibility in the latter [37].

- Taking a central-planner point of view likely overestimates the extent to which a BSP would use wind assets as upward reserves when he is forced to curtail part of the 
available wind power on very windy days with low demand. Indeed, when market rules allow it, he might hold back the curtailed volume in case his own portfolio needs inexpensive upward rebalancing.

For the stylized case studies, we assume a centrally cleared and perfectly competitive energy-reserve market. In addition to the practical challenges and considerations discussed above, we highlight some notable modelling assumptions below:

- We assume joint energy-reserve markets, in which energy and reserves clear simultaneously. Joint clearing exist in all of the competitive US markets (i.e., PJM, CAISO, RCOT, MISO and NYISO [39]), but only in a few European markets (e.g. Greece, Hungary, Ireland, Italy, Northern Ireland and Poland [11]). The majority of the ancillary services and electric energy market in Europe are cleared sequentially and in that order. Ideally, European reserve markets would share the temporal granularity of electricity markets, which would maximize the arbitrage opportunities between energy and reserve markets, and possibly approach the cost-efficiency of joint energy-reserve markets. However, accurately accounting for the arbitrage opportunities between energy and reserve markets is not within the scope of this work.

- Wind power is assumed to be the only source of uncertainty. When adopting daily but highly dynamic sizing (cases 4 and 5), the quarter-hourly reserve requirements nearly double towards the second half of the day. Solar power forecast errors, another important source of uncertainty associated with intermittent RES, are statistically independent from wind power forecast errors [22]. Therefore, including solar power uncertainty in the analysis is expected to amplify the cost benefits of 
reduced reserve requirements closer to the moment of delivery. Furthermore, higher resolution reserve products and dynamic sizing could enable the system operator to take into account that solar power uncertainty only exists during daylight, and procure less reserves outside that period. Including other sources of uncertainty that are less dependent on forecast lead times (e.g., load and unplanned outages) would flatten the reserve requirements and could reduce the cost benefits of frequent and dynamic sizing.

- Frequent reserve sizing would also allow incorporating other elements of the uncertainty characterization. For example, wind power forecast errors are shown to depend on the forecast itself, which is more reliable closer to real time [40]. More advanced dynamic reserve sizing methodologies might consider such additional features to construct PDFs that are optimally suited to the anticipated system conditions. De Vos et al. [3] propose the application of machine learning techniques to identify a cluster of $\mathrm{K}$ historically observed real-time imbalances that occurred under similar circumstances, as characterized by a given set of features (e.g., timeof-day, forecasted level of wind and solar power and residual load). Out of these $\mathrm{K}$ observed imbalances, a PDF is composed, from which the upward and downward reserve requirements can be determined based on a probabilistic reliability criterion (Fig. 3). Such additional features were not considered in this work, but may further inflate the benefits of more frequent sizing.

- Transmission system constraints are not considered in our analysis. The Belgian power system, which forms the basis for our case study, however, does not exhibit structural congestion: redispatching to alleviate transmission congestion affects 
$0.08 \%$ of the yearly electricity production and increases the annual operating cost by approximately $0.3 \%$ (2.9 million EUR per year) [41]. Therefore, we opted not to include transmission constraints in our analysis, as we did in in [42],[43], without significantly affecting the conclusions of this case study.

- Finally, other reserve providers, such as battery storage and active demand response, were not considered. These have advantages similar to those associated with non-spinning reserve providers, and hence have the potential to reduce the cost of procuring reserves [42],[43]. In addition, advanced scheduling procedures may allow "shifting" reserve requirements in time [44].

Despite these potential improvements to our analysis, the policy implication of this work is clear: implement shorter term, higher resolution reserve markets, carefully balancing the expected benefits with potential direct and indirect costs induced by the implementation and operation of these markets. Note that such reserve capacity markets may be implemented by each transmission system operator individually, i.e., without moving to joint, European energy-reserve capacity markets, which would require more fundamental changes to the European market clearing algorithm [45]. Our work supports recommendations put forward in Article 32 of Commission Regulation (EU) 2017/2195 [46], encouraging reserve procurement on a short-term basis (close to delivery and with high-resolution products) to the extent possible and where economically efficient. Elia, the Belgian TSO, has already demonstrated that dynamic dimensioning ensures reliability while reducing the cost of balancing compared with static dimensioning [47]. 


\section{Conclusion}

The large-scale introduction of variable and limitedly predictable renewables, such as wind power, increases the importance of markets for flexibility and requires energy market design changes.

In this work, we leverage a unit commitment and economic dispatch model to quantify the benefits of increasing the temporal granularity of reserve markets on the total operating cost and reserve market scarcity. Results of two realistic case studies revealed that the total operating cost savings reach $1.5 \%$ to $1.8 \%$ when moving from a very conservative to a highly dynamic joint energy-reserve and balancing market. Equally, adopting higher temporal granularities mitigated reserve market scarcity. Through varying each of the four considered temporal aspects separately, we isolated their impact. More frequent reserve sizing with a higher reserve resolution resulted in total cost-savings of $0.75-1.10 \%$. Reducing the reserve procurement contract duration and procuring reserve capacity more frequently yielded cost savings of $0.75 \%$ and facilitated the integration of intermittent renewables in reserve markets.

From a practical viewpoint, more frequent reserve sizing and procurement could pose challenges related to market operation, potentially triggering direct and indirect costs. These costs should be carefully monitored and balanced with the benefits high reserve capacity markets with a high temporal granularity. Furthermore, when moving towards a $100 \%$ renewable energy system, it is crucial that the integration of these RES is not hampered by lagging market design changes. The findings presented in this paper support the Belgian Transmission System Operator's assertion that dynamic dimensioning reduces the cost of balancing while safeguarding reliability [47], as well as 
the European Commission's recommendation to procure reserves on a short-term basis [46].

\section{Acknowledgements}

The research leading to this work was being carried out as a part of the EU-SysFlex project (Pan-European system with an efficient coordinated use of flexibilities for the integration of a large share of RES), which received funding from the European Union's Horizon 2020 research and innovation programme under grant agreement No 773505 . K. Bruninx is a post-doctoral research fellow of the Research Foundation - Flanders (FWO) at the University of Leuven and EnergyVille (mandate no. 12J3320N). The computational resources and services used in this work were provided by the VSC (Flemish Supercomputer Center), funded by the Research Foundation - Flanders (FWO) and the Flemish Government.

\section{References}

[1] K. Van den Bergh, R. B. Hytowitz, K. Bruninx, E. Delarue, W. D’haeseleer, and B. F. Hobbs, "Benefits of coordinating sizing, allocation and activation of reserves among market zones," Electr. Power Syst. Res., vol. 143, pp. 140-148, Feb. 2017.

[2] O. Borne, Y. Perez, and M. Petit, "Market integration or bids granularity to enhance flexibility provision by batteries of electric vehicles," Energy Policy, vol. 119, no. March, pp. 140-148, 2018.

[3] K. De Vos, N. Stevens, O. Devolder, A. Papavasiliou, B. Hebb, and J. Matthys-donnadieu, "Dynamic dimensioning approach for operating reserves : Proof of concept in Belgium," Energy Policy, vol. 124, pp. 272-285, 2019.

[4] A. Tuohy, P. Meibom, E. Denny, M. O'Malley, "Unit commitment for systems with significant wind penetration", IEEE Trans. Power Syst., vol. 24, no. 2, pp. 592-601, May 2009. 
[5] M. Hermans, K. Bruninx, E. Delarue. "Impact of CCGT Start-Up Flexibility and Cycling Costs Toward Renewables Integration," IEEE Trans. Sust. Energy, vol. 9, no. 3, pp. 1468-1476, 2018.

[6] A. M. Jafari, H. Zareipour, A. Schellenberg, N. Amjady, "The value of intra-day markets in power systems with high wind power penetration", IEEE Trans. Power Syst., vol. 29, no. 3, pp. 1121-1132, May 2014.

[7] T. Schulze, K. Mckinnon, "The value of stochastic programming in day-ahead and intra-day generation unit commitment", Energy, vol. 101, pp. 592-605, 2016.

[8] M. Bucksteeg, L. Nielsen, C. Weber, "Impacts of dynamic probabilistic reserve sizing techniques on reserve requirements and system costs", IEEE Trans. Sust. Energy, vol. 7, no. 4, pp. 1408-1420, 2016.

[9] B. Dallinger, H. Auer, G. Lettner, "Impact of harmonised common balancing capacity procurement in selected Central European electricity balancing markets", Applied Energy, vol. 222, pp. 351-368, 2018.

[10] S. Stoft, Power Systems Economics: Designing Markets for Electricity, IEEE Press, John Wiley and Sons, 2002.

[11] ENTSO-E, 2018. Survey on ancillary services procurement, balancing market design 2017. Present. by ENTSO-E Work. Gr. Ancillary Serv.

[12] J. Boucher, Y. Smeers, "Alternative models of restructured electricity systems part 1: No market power", Oper. Res., vol. 49, no. 6, pp. 821-838, 2001.

[13] C. Metzler, B. F. Hobbs, J. S. Pang, "Nash-Cournot equilibria in power markets on a linearized DC network with arbitrage: Formulations and properties", Netw. Spatial Econ., vol. 3, no. 2, pp. 123-150, 2003.

[14] K. Van den Bergh, K. Bruninx, E. Delarue, W. D’haeseleer, "LUSYM: A unit commitment model formulated as a mixed-integer linear program," Nov. 2016, [online] Available: https://www.mech.kuleuven.be/en/tme/research/energy_environment/Pdf/wpen2014-07-2.pdf

[15] K. Bruninx and E. Delarue, "Endogenous probabilistic reserve sizing and allocation in unit commitment models: Cost-effective, reliable and fast," IEEE Trans. Power Syst., vol. 32, no. 4, pp. 2593-2603, Jul. 2017. 
[16] E. Bakirtzis, P. Biskas, D. Labridis, A. Bakirtzis, "Multiple time resolution unit commitment for shortterm operations scheduling under high renewable penetration", IEEE Trans. Power Syst., vol. 29, no. 1, pp. 149-159, Jan. 2014.

[17] E. Ela, M. O'Malley, "Studying the variability and uncertainty impacts of variable generation at multiple timescales", IEEE Trans. Power Syst., vol. 27, no. 3, pp. 1324-1333, Aug. 2012.

[18] B. Ummels, M. Gibescu, E. Pelgrum, W. Kling, A. Brand, "Impacts of wind power on thermal generation unit commitment and dispatch", IEEE Trans. Energy Convers., vol. 22, no. 1, pp. 44-51, Mar. 2007.

[19] E. Bakirtzis, C. Simoglou, P. Biskas, D. Labridis, A. Bakirtzis, "Comparison of advanced power system operations models for large-scale renewable integration", Electr. Power Syst. Res., vol. 118, pp. 9099, 2015.

[20] FERC Order No. 764. [Online]. Available at: www.ferc.gov/whatsnew/comm-meet/2012/062112/E3.pdf

[21] Commission regulation (EU) 2017/1485 of 2 August 2017 establishing a guideline on electricity transmission system operation, 2017, [online] Available: http://data.europa.eu/eli/reg/2017/1485/oj

[22] K. Bruninx, "Improved Modeling of Unit Commitment Decisions Under Uncertainty," Ph.D. dissertation, pp. 150-151, KU Leuven, 2016.

[23] M. Hermans, K. Bruninx, E. Delarue, “On the Clearing Frequency of Joint Energy-Operating Reserve Markets and Generator Start-up Flexibility", 2019, [online] Available: https://www.mech.kuleuven.be/en/tme/research/energy_environment/Pdf/wp-en2019-08

[24] Grid Data, Elia, 2019. [Online]. Available: www.elia.be/en/grid-data.

[25] Deliverable 2.2: EU-SysFlex Scenarios and Network Sensitivities, 2018, [online] Available: https://eusysflex.com/first-three-eu-sysflex-technical-deliverables-published

[26] A. Schröder, F. Kunz, J. Meiss et al., "Data documentation-Current and prospective costs of electricity generation until 2050", 2013.

[27] D. Lunn and D. Roberts, "Technical assessment of the operation of coal \& gas fired plants," Parsons Brinckerhoff, 2014.

[28] Agora Energiewende, "Flexibility in thermal power plants," p. 116, 2017. 
[29] J. Keppler and M. Commetto, “Nuclear Energy and Renewables System Effects in Low-carbon Electricity Systems," NEA, 2012.

[30] C. Ruchti, H. Olia, K. Franitza, A. Ehrsam, W. Bauver, "Combined Cycle Power Plants as Ideal Solution to Balance Grid Fluctuations-Fast Start-up Capabilities", 43th Colloquium of Power Plant Technology Dresden Germany, Sep. 2011.

[31] H. Emberger, D. Hofmann, C. Kolk, "Economic Evaluation of Cycling Plants-An Approach to Show the Value of Operational Flexibility," Power-Gen Europe, 2006.

[32] L. Balling, "Flexible future for combined cycle", Mod. Power Syst., vol. 30, pp. 61-63, Dec. 2010.

[33] H. Chandler, "Harnessing Variable Renewables: A Guide to the Balancing Challenge," IEA, May 2011.

[34] Intertek APTECH, “Power Plant Cycling Costs," Nov. 2012.

[35] ENTSO-E, “Data portal-Statistical database," 2016. [Online]. Available: www.entsoe.eu/data/dataportal

[36] Elia, Explanatory Note on the ELIA LFC Block Operational Agreement, 2019, [online] Available: https://www.elia.be/en/products-and-services/balance/balancing-mechanism

[37] Elia, Study on the evolution towards a daily procurement of mFRR, 2018

[38] K. Poplavskaya, J. Lago, L. de Vries, “Effect of market design on strategic bidding behavior: Modelbased analysis of European electricity balancing markets," Applied Energy, vol. 270, art. no. 115130, 2020.

[39] P. González, J. Villar, C. A. Díaz, F. A. Campos, "Joint energy and reserve markets: Current implementations and modeling trends", Elect. Power Syst. Res., vol. 109, pp. 101-111, Apr. 2014.

[40] K. Bruninx, E. Delarue, "A statistical description of the error on wind power forecasts for probabilistic reserve sizing", IEEE Trans. Sustain. Energy, vol. 5, no. 3, pp. 995-1002, Jul. 2014.

[41] K. Van den Bergh, D. Couckuyt, E. Delarue, and W. D’haeseleer, “Redispatching in an interconnected electricity system with high renewables penetration," Electric Power System Research, vol. 127, no. 10, pp. 64-72, 2015.

[42] K. Bruninx, Y. Dvorkin, E. Delarue, H. Pandžić, W. D’haeseleer and D. S. Kirschen, "Coupling Pumped Hydro Energy Storage With Unit Commitment," in IEEE Transactions on Sustainable Energy, vol. 7, no. 2, pp. 786-796, April 2016. 
[43] K. Bruninx, Y. Dvorkin, E. Delarue, W. D’haeseleer and D. S. Kirschen, "Valuing Demand Response Controllability via Chance Constrained Programming," in IEEE Transactions on Sustainable Energy, vol. 9, no. 1, pp. 178-187, Jan. 2018.

[44] K. Bruninx, and E. Delarue, "Improved Energy Storage System \& Unit Commitment Scheduling," PowerTech 2017, June 19-22, 2017, Manchester, UK.

[45] N.E. Koltsaklis, A.S., Dagoumas, "Incorporating unit commitment aspects to the European electricity markets algorithm: An optimization model for the joint clearing of energy and reserve markets," Applied Energy, vol. 231, pp. 235-258, 2018.

[46] Commission regulation (EU) 2017/2195 of 23 November 2017 establishing a guideline on electricity balancing, 2017, [online] Available: http://data.europa.eu/eli/reg/2017/2195/oj

[47] Elia, Study on dynamic dimensioning of the FRR needs, 2017. 